\title{
ENTROPY AND RENORMALIZED SOLUTIONS FOR THE $p(x)$-LAPLACIAN EQUATION WITH MEASURE DATA
}

\author{
CHAO ZHANG ${ }^{\bowtie}$ and SHULIN ZHOU
}

(Received 24 June 2009)

\begin{abstract}
In this paper we prove the existence and uniqueness of both entropy solutions and renormalized solutions for the $p(x)$-Laplacian equation with variable exponents and a signed measure in $L^{1}(\Omega)+W^{-1, p^{\prime}(\cdot)}(\Omega)$. Moreover, we obtain the equivalence of entropy solutions and renormalized solutions.
\end{abstract}

2000 Mathematics subject classification: primary 35J70; secondary 35D05, 35D10, 46E35.

Keywords and phrases: variable exponents, entropy solutions, renormalized solutions, existence, uniqueness.

\section{Introduction}

Let $\Omega$ be a bounded open domain in $\mathbb{R}^{N}(N \geq 2)$ with Lipschitz boundary $\partial \Omega$. In this paper we study the nonlinear elliptic problem

$$
\begin{cases}-\operatorname{div}\left(|\nabla u|^{p(x)-2} \nabla u\right)=\mu & \text { in } \Omega \\ u=0 & \text { on } \partial \Omega\end{cases}
$$

where the variable exponent $p: \bar{\Omega} \rightarrow(1, \infty)$ is a continuous function, and $\mu$ is a signed measure in $L^{1}(\Omega)+W^{-1, p^{\prime}(\cdot)}(\Omega)$.

The study of differential equations and variational problems with nonstandard growth conditions has aroused much interest owing to the development of elastic mechanics, electro-rheological fluid dynamics and image processing, and so on. We refer the reader to $[13,30,31,34]$ and the references therein. The $p(x)$-growth conditions can be regarded as a very important class of nonstandard $(p, q)$-growth conditions. There are already numerous results for such kinds of problems (see $[1-3,5,16,17,20])$. The functional spaces used to deal with these problems are the generalized Lebesgue spaces $L^{p(x)}(\Omega)$ and the generalized Lebesgue-Sobolev spaces $W^{k, p(x)}(\Omega)$.

This work was supported in part by the NBRPC under Grant 2006CB705700 and the NSFC under Grant 10990013.

(C) 2010 Australian Mathematical Publishing Association Inc. 0004-9727/2010 \$16.00 
Under our assumptions, it is reasonable to work with entropy solutions or renormalized solutions, which need less regularity than the usual weak solutions. The notion of renormalized solutions was first introduced by DiPerna and Lions [15] for the study of the Boltzmann equation. It was then adapted to the study of some nonlinear elliptic or parabolic problems and evolution problems in fluid mechanics. We refer the reader to $[7-9,12,27]$ for details. At the same time the notion of entropy solutions was proposed by Bénilan et al. [6] for nonlinear elliptic problems. This framework was extended to related problems with constant $p$ in $[4,10,11,29]$. The interesting and difficult cases are those of $1<p \leq N$, since the variational methods of LerayLions (see [26]) can be easily applied for $p>N$, with the solution being bounded and its gradient being in $L^{p}(\Omega)$.

Recently, Sanchón and Urbano [32] studied a Dirichlet problem of the $p(x)$-Laplace equation and obtained the existence and uniqueness of entropy solutions for $L^{1}$ data, as well as integrability results for the solution and its gradient. The proofs rely crucially on a priori estimates in Marcinkiewicz spaces with variable exponents.

The aim of this paper is to extend the results in [6, 11,32], taking into account a signed measure $\mu$ in $L^{1}(\Omega)+W^{-1, p^{\prime}(\cdot)}(\Omega)$. As far as we know, there are few papers concerned with the nonlinear elliptic or parabolic equations involving measure data and multiple anisotropic exponents. Therefore, the study of problem (1.1) is a new and interesting topic. The uniqueness of the entropy solutions and renormalized solutions will strongly rely on the structure of the measure $\mu$, that is, $\mu$ belongs to $L^{1}(\Omega)+$ $W^{-1, p^{\prime}(\cdot)}(\Omega)$. If not, there is a counterexample in [11] showing why the definition of the entropy solution is not suitable for uniqueness if the measure $\mu$ is the Dirac mass.

Inspired by [6, 11, 25], we develop a refined method which is different from [32]. We first construct an approximate solution sequence for problem (1.1) and establish some a priori estimates. Next, we draw a subsequence to obtain a limit function, and prove this function is an entropy solution. The advantage of our method is that we can give the concrete expression of the limit function of the approximate solutions by means of truncation techniques and prove the strong convergence of the truncations of the approximate solutions. Based on this fact, we obtain that the entropy solution of problem (1.1) is also a renormalized solution. It is worth pointing out that we generalize the definition of renormalized solutions from $L^{1}$ functions to measurable functions. This allows us to drop some redundant conditions imposed on the exponent $p(x)$ in the previous work. Even for the constant exponent case, to the best of our knowledge, there seems to be no result about the existence and uniqueness of renormalized solutions to such elliptic equations with a signed measure $\mu$ in $L^{1}(\Omega)+W^{-1, p^{\prime}}(\Omega)$. By choosing suitable test functions and employing the decomposition for the region of integration according to the different contributions corresponding to different integration sets, we prove the uniqueness of the entropy solutions and renormalized solutions, and thus the equivalence of the entropy solutions and renormalized solutions.

For the convenience of the reader, we recall some definitions and basic properties of the generalized Lebesgue spaces $L^{p(x)}(\Omega)$ and the generalized Lebesgue-Sobolev spaces $W^{k, p(x)}(\Omega)$. 
Set $C_{+}(\bar{\Omega})=\left\{h \in C(\bar{\Omega}): \min _{x \in \bar{\Omega}} h(x)>1\right\}$. For any $h \in C_{+}(\bar{\Omega})$ we define

$$
h_{+}=\sup _{x \in \Omega} h(x) \quad \text { and } \quad h_{-}=\inf _{x \in \Omega} h(x) .
$$

For any $p \in C_{+}(\bar{\Omega})$, we introduce the variable exponent Lebesgue space $L^{p(\cdot)}(\Omega)$ to consist of all measurable functions such that

$$
\int_{\Omega}|u(x)|^{p(x)} d x<\infty,
$$

endowed with the Luxemburg norm

$$
|u|_{p(\cdot)}=\inf \left\{\lambda>0: \int_{\Omega}\left|\frac{u(x)}{\lambda}\right|^{p(x)} d x \leq 1\right\},
$$

which is a separable and reflexive Banach space. The dual space of $L^{p(x)}(\Omega)$ is $L^{p^{\prime}(x)}(\Omega)$, where $1 / p(x)+1 / p^{\prime}(x)=1$. If $p(x)$ is a constant function, then the variable exponent Lebesgue space coincides with the classical Lebesgue space. The variable exponent Lebesgue space is a special case of the Orlicz-Musielak space treated by Musielak [28].

For any positive integer $k$, denote

$$
W^{k, p(x)}(\Omega)=\left\{u \in L^{p(x)}(\Omega): D^{\alpha} u \in L^{p(x)}(\Omega),|\alpha| \leq k\right\},
$$

where the norm is defined as

$$
\|u\|_{W^{k, p(x)}(\Omega)}=\sum_{|\alpha| \leq k}\left|D^{\alpha} u\right|_{p(x)} .
$$

Here $W^{k, p(x)}(\Omega)$ is also a Banach space. It is called a generalized Lebesgue-Sobolev space, which is a special generalized Orlicz-Sobolev space. An interesting feature of a generalized Lebesgue-Sobolev space is that smooth functions are not dense in this space without additional assumptions on the exponent $p(x)$. This was observed by Zhikov [33] in connection with the Lavrentiev phenomenon. However, when the exponent $p(x)$ is log-Hölder continuous, that is, there is a constant $C$ such that

$$
|p(x)-p(y)| \leq \frac{C}{-\log |x-y|}
$$

for every $x, y \in \Omega$ with $|x-y| \leq 1 / 2$, then smooth functions are dense in variable exponent Sobolev spaces and there is no confusion in defining the Sobolev space with zero boundary values, $W_{0}^{1, p(\cdot)}(\Omega)$, as the completion of $C_{0}^{\infty}(\Omega)$ with respect to the norm $\|u\|_{W^{1, p(\cdot)(\Omega)}}($ see [21]).

Throughout this paper we assume that $p(x) \in C_{+}(\bar{\Omega})$ with $1<p_{-} \leq p_{+}<N$ satisfies the log-Hölder continuity condition (1.2), and $\mu$ is a signed measure in $L^{1}(\Omega)+W^{-1, p^{\prime}(\cdot)}(\Omega)$, that is,

$$
\mu=f-\operatorname{div} F
$$


where $f \in L^{1}(\Omega)$ and $F \in\left(L^{p^{\prime}(\cdot)}(\Omega)\right)^{N}$. Let $T_{k}$ denote the truncation function at height $k \geq 0$ :

$$
T_{k}(r)=\min \{k, \max \{r,-k\}\}= \begin{cases}k & \text { if } r \geq k \\ r & \text { if }|r|<k \\ -k & \text { if } r \leq-k\end{cases}
$$

We denote

$$
\mathcal{T}_{0}^{1, p(\cdot)}(\Omega)=\left\{u: \Omega \rightarrow \mathbb{R} \text { is measurable } \mid T_{k}(u) \in W_{0}^{1, p(\cdot)}(\Omega), \text { for every } k>0\right\} .
$$

Next we define the very weak gradient of a measurable function $u \in \mathcal{T}_{0}^{1, p(\cdot)}(\Omega)$. The proof follows from [6, Lemma 2.1] due to the fact that $W_{0}^{1, p(\cdot)}(\Omega) \subset W_{0}^{1, p_{-}}(\Omega)$.

Proposition 1.1. For every measurable function $u \in \mathcal{T}_{0}^{1, p(\cdot)}(\Omega)$, there exists a unique measurable function $v: \Omega \rightarrow \mathbb{R}^{N}$, which we call the very weak gradient of $u$ and denote $v=\nabla u$, such that

$$
\nabla T_{k}(u)=v \chi_{\{|u|<k\}} \quad \text { for almost every } x \in \Omega \text { and for every } k>0,
$$

where $\chi_{E}$ denotes the characteristic function of a measurable set $E$. Moreover, if $u$ belongs to $W_{0}^{1,1}(\Omega)$, then $v$ coincides with the weak gradient of $u$.

The notion of the very weak gradient allows us to give the following definitions of entropy solutions and renormalized solutions for problem (1.1).

Definition 1.2. A measurable function $u \in \mathcal{T}_{0}^{1, p(\cdot)}(\Omega)$ is an entropy solution to problem (1.1) if

$$
\int_{\Omega}|\nabla u|^{p(x)-2} \nabla u \cdot \nabla T_{k}(u-\phi) d x=\int_{\Omega} f T_{k}(u-\phi) d x+\int_{\Omega} F \cdot \nabla T_{k}(u-\phi) d x,
$$

for all $\phi \in W_{0}^{1, p(\cdot)}(\Omega) \cap L^{\infty}(\Omega)$.

DEFINITION 1.3. A measurable function $u \in \mathcal{T}_{0}^{1, p(\cdot)}(\Omega)$ is a renormalized solution to problem (1.1) if the following conditions are satisfied:

(i) $\lim _{n \rightarrow \infty} \int_{\{n \leq|u| \leq n+1\}}|\nabla u|^{p(x)} d x=0$;

(ii) for every function $\varphi \in C_{0}^{\infty}(\Omega)$ and $S \in W^{1, \infty}(\mathbb{R})$ such that $S$ has compact support,

$$
\begin{gathered}
\int_{\Omega}\left[S(u)|\nabla u|^{p(x)-2} \nabla u \cdot \nabla \varphi+S^{\prime}(u)|\nabla u|^{p(x)} \varphi\right] d x \\
=\int_{\Omega}[f S(u) \varphi+F \cdot \nabla(S(u) \varphi)] d x
\end{gathered}
$$

holds. 
Now we state our main results as follows.

THEOREM 1.4. Assume that $\mu$ satisfies (1.3). Then there exists a unique entropy solution $u \in \mathcal{T}_{0}^{1, p(\cdot)}(\Omega)$ for problem (1.1).

THEOREM 1.5. Assume that $\mu$ satisfies (1.3). Then the entropy solution $u$ in Theorem 1.4 is also a renormalized solution for problem (1.1). Moreover, the renormalized solution is unique.

REMARK 1.6. The entropy solution for problem (1.1) is equivalent to the renormalized solution for problem (1.1).

Given two bounded measurable functions $p(\cdot), q(\cdot): \Omega \rightarrow \mathbb{R}$, we write

$$
q(\cdot) \ll p(\cdot) \quad \text { if }(p-q)_{-}>0 .
$$

REMARK 1.7. If $u$ is an entropy solution or renormalized solution of problem (1.1) and $p(\cdot) \gg 2-1 / N$, then, following the arguments in [32] by some necessary changes and using some a priori estimates in Marcinkiewicz for $u$ and $\nabla u$, we can obtain that $u \in L^{q(\cdot)}(\Omega)$, for all $1 \ll q(\cdot) \ll q_{0}(\cdot)$, and $u \in W_{0}^{1, q(\cdot)}(\Omega)$, for all $1 \ll q(\cdot) \ll q_{1}(\cdot)$, where

$$
q_{0}(\cdot):=\frac{p^{*}(\cdot)}{\left(p^{\prime}\right)_{+}} \quad \text { and } \quad q_{1}(\cdot):=\frac{q_{0}(\cdot)}{q_{0}(\cdot)+1} p(\cdot) .
$$

The rest of this paper is organized as follows. In Section 2, we state some basic results that will be used later. We will prove the main results in Section 3. In the following sections $C$ will represent a generic constant that may change from line to line even if in the same inequality.

\section{Preliminaries}

In this section, we first state some elementary results for the generalized Lebesgue spaces $L^{p(x)}(\Omega)$ and the generalized Lebesgue-Sobolev spaces $W^{k, p(x)}(\Omega)$.

\section{LEMMA 2.1 [20, 24].}

(1) The space $L^{p(\cdot)}(\Omega)$ is a separable, uniform convex Banach space, and its conjugate space is $L^{p^{\prime}(\cdot)}(\Omega)$ where $1 / p(x)+1 / p^{\prime}(x)=1$. For any $u \in L^{p(\cdot)}(\Omega)$ and $v \in L^{p^{\prime}(\cdot)}(\Omega)$,

$$
\left|\int_{\Omega} u v d x\right| \leq\left(\frac{1}{p_{-}}+\frac{1}{\left(p^{\prime}\right)_{-}}\right)|u|_{p(x)}|v|_{p^{\prime}(x)} \leq 2|u|_{p(x)}|v|_{p^{\prime}(x)} .
$$

(2) If $p_{1}, p_{2} \in C_{+}(\bar{\Omega}), p_{1}(x) \leq p_{2}(x)$ for any $x \in \Omega$, then there exists the continuous embedding $L^{p_{2}(x)}(\Omega) \hookrightarrow L^{p_{1}(x)}(\Omega)$, whose norm does not exceed $|\Omega|+1$. 
LEMMA 2.2 [20]. If we denote

$$
\rho(u)=\int_{\Omega}|u|^{p(x)} d x, \quad \forall u \in L^{p(x)}(\Omega),
$$

then

$$
\min \left\{|u|_{p(x)}^{p_{-}},|u|_{p(x)}^{p_{+}}\right\} \leq \rho(u) \leq \max \left\{|u|_{p(x)}^{p_{-}},|u|_{p(x)}^{p_{+}}\right\} .
$$

LEMMA 2.3 [20]. Let $p \in C_{+}(\bar{\Omega})$. Then $W^{k, p(x)}(\Omega)$ is a separable and reflexive Banach space.

Lemma $2.4[23,24]$. Let $p \in C_{+}(\bar{\Omega})$. Then, for $u \in W_{0}^{1, p(\cdot)}(\Omega)$, the $p(\cdot)$-Poincaré inequality

$$
|u|_{p(x)} \leq C|\nabla u|_{p(x)}
$$

holds, where the positive constant $C$ depends on $p$ and $\Omega$.

LEMMA $2.5[14,19]$. Let $\Omega \subset \mathbb{R}^{N}$ be an open, bounded set with Lipschitz boundary and let $p(x) \in C_{+}(\bar{\Omega})$ with $1<p_{-} \leq p_{+}<N$ satisfy the log-Hölder continuity condition (1.2). If $q \in L^{\infty}(\Omega)$ with $q_{-}>1$ satisfies

$$
q(x) \leq p^{*}(x):=\frac{N p(x)}{N-p(x)}, \quad \forall x \in \Omega,
$$

then

$$
W^{1, p(x)}(\Omega) \hookrightarrow L^{q(x)}(\Omega)
$$

and the imbedding is compact if $\inf _{x \in \Omega}\left(p^{*}(x)-q(x)\right)>0$.

Now we begin to prove some a priori important estimates for entropy solutions of problem (1.1).

PROPOSITION 2.6. If $u$ is an entropy solution of problem (1.1), then there exists a positive constant $C$ such that, for all $k>1$,

$$
\operatorname{meas}\{|u|>k\} \leq \frac{C(M+1)^{\left(p^{*}\right)_{-} / p_{-}}}{k^{\left(p^{*}\right)_{-}\left(1-\left(1 / p_{-}\right)\right)}}
$$

holds, where

$$
M=\frac{p_{-}}{p_{-}-1}\|f\|_{L^{1}(\Omega)}+\frac{p_{-}\left(p_{+}-1\right)}{p_{+}\left(p_{-}-1\right)} \int_{\Omega}|F|^{p^{\prime}(x)} d x .
$$

PROOF. Choosing $\phi=0$ in the entropy equality (1.4) and using Young's inequality, we obtain

$$
\begin{aligned}
\int_{\Omega}\left|\nabla T_{k}(u)\right|^{p(x)} d x & =\int_{\{|u| \leq k\}}|\nabla u|^{p(x)} d x \\
& \leq \frac{k p_{-}}{p_{-}-1}\|f\|_{L^{1}(\Omega)}+\frac{p_{-}\left(p_{+}-1\right)}{p_{+}\left(p_{-}-1\right)} \int_{\Omega}|F|^{p^{\prime}(x)} d x,
\end{aligned}
$$


which implies that, for all $k>1$,

$$
\frac{1}{k} \int_{\Omega}\left|\nabla T_{k}(u)\right|^{p(x)} d x \leq M_{1}+\frac{M_{2}}{k} \leq M:=M_{1}+M_{2},
$$

where

$$
M_{1}=\frac{p_{-}}{p_{-}-1}\|f\|_{L^{1}(\Omega)}, \quad M_{2}=\frac{p_{-}\left(p_{+}-1\right)}{p_{+}\left(p_{-}-1\right)} \int_{\Omega}|F|^{p^{\prime}(x)} d x .
$$

Recalling the Sobolev embedding theorem in Lemmas 2.5 and 2.1, we have the continuous embedding

$$
W_{0}^{1, p(x)}(\Omega) \hookrightarrow L^{p^{*}(x)}(\Omega) \hookrightarrow L^{\left(p^{*}\right)-}(\Omega),
$$

where $p^{*}(x)=(N p(x) / N-p(x))$ and $\left(p^{*}\right)_{-}=\left(N p_{-} / N-p_{-}\right)$. It follows from Lemma 2.2 and (2.1) that, for every $k>1$,

$$
\begin{aligned}
\left\|T_{k}(u)\right\|_{\left(p^{*}\right)_{-}} & =\left|T_{k}(u)\right|_{\left(p^{*}\right)_{-}} \leq C\left|\nabla T_{k}(u)\right|_{p(x)} \\
& \leq C\left(\int_{\Omega}\left|\nabla T_{k}(u)\right|^{p(x)} d x\right)^{\beta} \leq C(M k)^{\beta},
\end{aligned}
$$

where

$$
\beta= \begin{cases}\frac{1}{p_{-}} & \text {if }\left|\nabla T_{k}(u)\right|_{p(\cdot)} \geq 1, \\ \frac{1}{p_{+}} & \text {if }\left|\nabla T_{k}(u)\right|_{p(\cdot)} \leq 1 .\end{cases}
$$

Noting that $\{|u| \geq k\}=\left\{\left|T_{k}(u)\right| \geq k\right\}$,

$$
\operatorname{meas}\{|u|>k\} \leq\left(\frac{\left\|T_{k}(u)\right\|_{\left(p^{*}\right)_{-}}}{k}\right)^{\left(p^{*}\right)_{-}} \leq \frac{C M^{\beta\left(p^{*}\right)_{-}}}{k^{\left(p^{*}\right)_{-}(1-\beta)}} \leq \frac{C(M+1)^{\left(p^{*}\right)_{-} / p_{-}}}{k^{\left(p^{*}\right)_{-}\left(1-\left(1 / p_{-}\right)\right)}} .
$$

This finishes the proof.

PROPOSITION 2.7. If $u$ is an entropy solution of problem (1.1), then, for a given $a>0$,

$$
\lim _{k \rightarrow \infty} \int_{\{k \leq|u| \leq k+a\}}|\nabla u|^{p(x)} d x=0 .
$$

PROOF. For given $a, k>0$, define the function $T_{k, a}(s)=T_{a}\left(s-T_{k}(s)\right)$ as

$$
T_{k, a}(s)= \begin{cases}s-k \operatorname{sign}(s) & \text { if } k \leq|s|<k+a \\ a \operatorname{sign}(s) & \text { if }|s| \geq k+a \\ 0 & \text { if }|s| \leq k\end{cases}
$$

Using $T_{k, a}(u)$ to take the place of $T_{k}(u-\phi)$ in (1.4), we find

$$
\int_{\{k \leq|u| \leq k+a\}}|\nabla u|^{p(x)-2} \nabla u \cdot \nabla u d x \leq \int_{\Omega} f T_{k, a}(u) d x+\int_{\{k \leq|u| \leq k+a\}} F \cdot \nabla u d x .
$$


In view of Young's inequality,

$$
\begin{aligned}
& \int_{\{k \leq|u| \leq k+a\}}|\nabla u|^{p(x)} d x \\
& \leq a \int_{\{|u|>k\}}|f| d x+\int_{\{k \leq|u| \leq k+a\}}\left(\frac{1}{p(x)}|\nabla u|^{p(x)}+\frac{p(x)-1}{p(x)}|F|^{p^{\prime}(x)}\right) d x \\
& \leq a \int_{\{|u|>k\}}|f| d x+\frac{1}{p_{-}} \int_{\{k \leq|u| \leq k+a\}}|\nabla u|^{p(x)} d x \\
&+\frac{p_{+}-1}{p_{+}} \int_{\{k \leq|u| \leq k+a\}}|F|^{p^{\prime}(x)} d x,
\end{aligned}
$$

which implies that

$$
\begin{aligned}
& \int_{\{k \leq|u| \leq k+a\}}|\nabla u|^{p(x)} d x \\
& \quad \leq \frac{a p_{-}}{p_{-}-1} \int_{\{|u|>k\}}|f| d x+\frac{p_{-}\left(p_{+}-1\right)}{p_{+}\left(p_{-}-1\right)} \int_{\{k \leq|u| \leq k+a\}}|F|^{p^{\prime}(x)} d x .
\end{aligned}
$$

Therefore, we obtain that

$$
\lim _{k \rightarrow \infty} \int_{\{k \leq|u| \leq k+a\}}|\nabla u|^{p(x)} d x=0 .
$$

This finishes the proof.

\section{The proofs of main results}

In this section we are ready to prove the main results. First we prove the existence and uniqueness of the entropy solutions for problem (1.1).

PROOF OF THEOREM 1.4. (1) Existence of entropy solutions. We first introduce the approximate problems. Find two sequences of $C_{0}^{\infty}(\Omega)$ functions $\left\{f_{n}\right\}$ and $\left\{F_{n}\right\}$ strongly converging respectively to $f$ in $L^{1}(\Omega)$ and to $F$ in $\left(L^{p^{\prime}(\cdot)}(\Omega)\right)^{N}$ such that

$$
\left\|f_{n}\right\|_{L^{1}(\Omega)} \leq\|f\|_{L^{1}(\Omega)}, \quad \int_{\Omega}\left|F_{n}\right|^{p^{\prime}(x)} d x \leq \int_{\Omega}|F|^{p^{\prime}(x)} d x .
$$

Then we consider the approximate problems of (1.1),

$$
\begin{cases}-\operatorname{div}\left(\left|\nabla u_{n}\right|^{p(x)-2} \nabla u_{n}\right)=f_{n}-\operatorname{div}\left(F_{n}\right) & \text { in } \Omega \\ u_{n}=0 & \text { on } \partial \Omega .\end{cases}
$$

By employing the variation methods and arguments in [18, Theorem 4.2] or [26], we easily find a unique weak solution $u_{n} \in W_{0}^{1, p(\cdot)}(\Omega)$ of problem (3.2), which is obviously an entropy solution. Our aim is to prove that a subsequence of these approximate solutions $\left\{u_{n}\right\}$ converges to a measurable function $u$, which is an entropy solution of problem (1.1). We will divide the proof into several steps. Some of the reasoning is based on the ideas developed in $[6,11,25]$. 
Choosing $T_{k}\left(u_{n}\right)$ as a test function in (3.2) and applying Young's inequality on the right-hand side,

$$
\begin{gathered}
\int_{\Omega}\left|\nabla T_{k}\left(u_{n}\right)\right|^{p(x)} d x \leq k\left\|f_{n}\right\|_{L^{1}(\Omega)}+\int_{\Omega} \frac{1}{p(x)}\left|\nabla T_{k}\left(u_{n}\right)\right|^{p(x)} d x \\
+\int_{\Omega} \frac{1}{p^{\prime}(x)}\left|F_{n}\right|^{p^{\prime}(x)} d x,
\end{gathered}
$$

which implies that

$$
\begin{aligned}
\int_{\Omega}\left|\nabla T_{k}\left(u_{n}\right)\right|^{p(x)} d x & \leq \frac{k p_{-}}{p_{-}-1}\left\|f_{n}\right\|_{L^{1}(\Omega)}+\frac{p_{-}\left(p_{+}-1\right)}{p_{+}\left(p_{-}-1\right)} \int_{\Omega}\left|F_{n}\right|^{p^{\prime}(x)} d x \\
& \leq \frac{k p_{-}}{p_{-}-1}\|f\|_{L^{1}(\Omega)}+\frac{p_{-}\left(p_{+}-1\right)}{p_{+}\left(p_{-}-1\right)} \int_{\Omega}|F|^{p^{\prime}(x)} d x .
\end{aligned}
$$

Step 1. Construct a subsequence $\left\{u^{n}\right\}$ of $\left\{u_{n}\right\}$ and a limit function $u$ such that, for every positive integer $k$,

$$
T_{k} u^{n} \rightarrow T_{k} u \quad \text { strongly in } L^{q}(\Omega),
$$

where $q=\left(p_{-}+\left(p^{*}\right)_{-}\right) / 2$.

As $\left\{T_{1} u_{n}\right\}$ is bounded in $W_{0}^{1, p(\cdot)}(\Omega)$ from (3.3), recalling Lemma 2.5, there exist a subsequence $\left\{u_{n}^{1}\right\}$ of $\left\{u_{n}\right\}$ and a function $v_{1} \in L^{q}(\Omega)$ with $\left|v_{1}\right| \leq 1$ such that

$$
T_{1} u_{n}^{1} \rightarrow v_{1} \quad \text { strongly in } L^{q}(\Omega) .
$$

As $\left\{T_{2} u_{n}^{1}\right\}$ is bounded in $W_{0}^{1, p(\cdot)}(\Omega)$, there exist a subsequence $\left\{u_{n}^{2}\right\}$ of $\left\{u_{n}^{1}\right\}$ and a function $v_{2} \in L^{q}(\Omega)$ with $\left|v_{2}\right| \leq 2$ such that

$$
T_{2} u_{n}^{2} \rightarrow v_{2} \quad \text { strongly in } L^{q}(\Omega) .
$$

By the same procedures, for every positive integer $k$, we find there exist a subsequence $\left\{u_{n}^{k}\right\}$ of $\left\{u_{n}^{k-1}\right\}$ and a function $v_{k} \in L^{q}(\Omega)$ with $\left|v_{k}\right| \leq k$ such that

$$
T_{k} u_{n}^{k} \rightarrow v_{k} \quad \text { strongly in } L^{q}(\Omega) .
$$

Set $u^{n}=u_{n}^{n}, n=1,2, \ldots$ Then we have, for every positive integer $k$,

$$
T_{k} u^{n} \rightarrow v_{k} \quad \text { strongly in } L^{q}(\Omega) .
$$

Let $l, k$ be any two positive integers with $l<k$. Noting the equality

$$
T_{l}\left(T_{k} u^{n}\right)=T_{l} u^{n},
$$

and sending $n \rightarrow \infty$, we conclude that

$$
T_{l} v_{k}=v_{l}
$$


It follows from the construction of $v_{k}$ that

$$
\begin{aligned}
\Omega & =\left\{0 \leq\left|v_{1}\right|<1\right\} \cup\left\{\left|v_{1}\right|=1\right\} \cup\{\text { a negligible set }\} \\
& =\left\{0 \leq\left|v_{1}\right|<1\right\} \cup\left\{1 \leq\left|v_{2}\right|<2\right\} \cup\left\{\left|v_{2}\right|=2\right\} \cup\{\text { a negligible set }\} \\
& =\cdots \\
& =\bigcup_{k=1}\left\{k-1 \leq\left|v_{k}\right|<k\right\} \bigcup\{\text { a negligible set }\} .
\end{aligned}
$$

Then we define a measurable function $u$ in $\Omega$ by

$$
u(x)= \begin{cases}v_{k}(x) & \text { when } k-1 \leq\left|v_{k}(x)\right|<k \text { for } k=1,2, \ldots \\ 0 & \text { a negligible set }\end{cases}
$$

which satisfies the condition

$$
T_{k} u=v_{k} \quad \text { for } k=1,2, \ldots
$$

Step 2. Prove the convergence in measure of $\left\{u^{n}\right\}$ and find its subsequence which is almost everywhere (a.e.) convergent in $\Omega$.

For every fixed $\epsilon>0$, and every positive integer $k$, we know that

$$
\left\{\left|u^{n}-u^{m}\right|>\epsilon\right\} \subset\left\{\left|u^{n}\right|>k\right\} \cup\left\{\left|u^{m}\right|>k\right\} \cup\left\{\left|T_{k}\left(u^{n}\right)-T_{k}\left(u^{m}\right)\right|>\epsilon\right\} .
$$

Recalling the convergence of $\left\{T_{k} u^{n}\right\}$ in $L^{q}(\Omega)$ and Proposition 2.6, we conclude that

$$
\limsup _{n, m \rightarrow \infty} \operatorname{meas}\left\{\left|u^{n}-u^{m}\right|>\epsilon\right\} \leq C\left(p(\cdot),\|f\|_{L^{1}(\Omega)},|F|_{p(\cdot)}\right) k^{-\alpha},
$$

where $\alpha=\left(p^{*}\right)_{-}\left(1-\left(1 / p_{-}\right)\right)>0$.

Because of the arbitrariness of $k$, we prove that

$$
\limsup _{n, m \rightarrow \infty} \operatorname{meas}\left\{\left|u^{n}-u^{m}\right|>\epsilon\right\}=0,
$$

which implies the convergence in measure of $\left\{u^{n}\right\}$, and then we find an a.e. convergent subsequence (still denoted by $\left\{u^{n}\right\}$ ) in $\Omega$ such that

$$
u^{n} \rightarrow u \quad \text { a.e. in } \Omega \text {. }
$$

In the following we denote $\left\{u^{n}\right\}$ by $\left\{u_{n}\right\}$ for simplicity of notation. $k>0$.

Step 3. We prove that $T_{k}\left(u_{n}\right)$ strongly converges to $T_{k}(u)$ in $W_{0}^{1, p(x)}(\Omega)$, for every

Since the set $\{(k, h): k, h=1,2, \ldots\}$ is countable, using the boundedness of $T_{k}\left(u_{n}\right)$ and $T_{2 k}\left(u_{n}-T_{h}\left(u_{n}\right)\right)$ in $W_{0}^{1, p(\cdot)}(\Omega)$ and the diagonal procedures, we draw a subsequence (still denoted by $\left\{u_{n}\right\}$ ) from $\left\{u_{n}\right\}$ such that, for every pair of positive 
integers $k, h$,

$$
\begin{gathered}
T_{k}\left(u_{n}\right) \rightarrow T_{k}(u) \quad \text { weakly in } W_{0}^{1, p(\cdot)}(\Omega), \\
T_{2 k}\left(u_{n}-T_{h}\left(u_{n}\right)\right) \rightarrow T_{2 k}\left(u-T_{h}(u)\right) \quad \text { weakly in } W_{0}^{1, p(\cdot)}(\Omega)
\end{gathered}
$$

and

$$
T_{2 k}\left(u_{n}-T_{h}\left(u_{n}\right)+T_{k}\left(u_{n}\right)-T_{k}(u)\right) \rightarrow T_{2 k}\left(u-T_{h}(u)\right) \quad \text { weakly in } W_{0}^{1, p(\cdot)}(\Omega) .
$$

First we prove that this subsequence $\left\{u_{n}\right\}$ has the property that $T_{k} u_{n}$ strongly converges to $T_{k} u$ in $W_{0}^{1, p(\cdot)}(\Omega)$ for every positive integer $k$.

Fix a positive integer $k$. Let $h$ be a positive integer satisfying $h>k$. We choose

$$
w_{n}=T_{2 k}\left(u_{n}-T_{h}\left(u_{n}\right)+T_{k}\left(u_{n}\right)-T_{k}(u)\right)
$$

as a test function in (3.2). If we set $M=4 k+h$, then it is easy to see that $\nabla w_{n}=0$ where $\left|u_{n}\right|>M$. Therefore, we may write the weak form of (3.2) as

$$
\int_{\Omega}\left|\nabla T_{M}\left(u_{n}\right)\right|^{p(x)-2} \nabla T_{M}\left(u_{n}\right) \cdot \nabla w_{n} d x=\int_{\Omega} f_{n} w_{n} d x+\int_{\Omega} F_{n} \cdot \nabla w_{n} d x .
$$

Splitting the integral on the left-hand side on the sets where $\left|u_{n}\right| \leq k$ and where $\left|u_{n}\right|>k$, and discarding some nonnegative terms, we find

$$
\begin{aligned}
\int_{\Omega} \mid \nabla & \left.T_{M}\left(u_{n}\right)\right|^{p(x)-2} \nabla T_{M}\left(u_{n}\right) \cdot \nabla T_{2 k}\left(u_{n}-T_{h}\left(u_{n}\right)+T_{k}\left(u_{n}\right)-T_{k}(u)\right) d x \\
\geq & \int_{\Omega}\left|\nabla T_{k}\left(u_{n}\right)\right|^{p(x)-2} \nabla T_{k}\left(u_{n}\right) \cdot \nabla\left(T_{k}\left(u_{n}\right)-T_{k}(u)\right) d x \\
& -\left.\int_{\left\{\left|u_{n}\right|>k\right\}}|| \nabla T_{M}\left(u_{n}\right)\right|^{p(x)-2} \nabla T_{M}\left(u_{n}\right)|| \nabla T_{k}(u) \mid d x .
\end{aligned}
$$

It follows from the above inequality that

$$
\begin{aligned}
\int_{\Omega}(\mid \nabla & \left.\left.T_{k}\left(u_{n}\right)\right|^{p(x)-2} \nabla T_{k}\left(u_{n}\right)-\left|\nabla T_{k}(u)\right|^{p(x)-2} \nabla T_{k}(u)\right) \cdot \nabla\left(T_{k}\left(u_{n}\right)-T_{k}(u)\right) d x \\
\leq & \left.\int_{\left\{\left|u_{n}\right|>k\right\}}|| \nabla T_{M}\left(u_{n}\right)\right|^{p(x)-2} \nabla T_{M}\left(u_{n}\right)|| \nabla T_{k}(u) \mid d x \\
& +\int_{\Omega} f_{n} T_{2 k}\left(u_{n}-T_{h}\left(u_{n}\right)+T_{k}\left(u_{n}\right)-T_{k}(u)\right) d x \\
& +\int_{\Omega} F_{n} \cdot \nabla T_{2 k}\left(u_{n}-T_{h}\left(u_{n}\right)+T_{k}\left(u_{n}\right)-T_{k}(u)\right) d x \\
& -\int_{\Omega}\left|\nabla T_{k}(u)\right|^{p(x)-2} \nabla T_{k}(u) \cdot \nabla\left(T_{k}\left(u_{n}\right)-T_{k}(u)\right) d x \\
& :=I_{1}+I_{2}+I_{3}+I_{4} .
\end{aligned}
$$


Using the properties of $L^{p(x)}(\Omega)$ and the similar estimates as in [25], we can show that the limits of $I_{1}, I_{2}, I_{3}$ and $I_{4}$ are zeros when $n$, and then $h$ tend to infinity, respectively.

Therefore, passing to the limits in (3.8) as $n$, and then $h$ tend to infinity, we deduce that

$$
\lim _{n \rightarrow+\infty} E(n)=0
$$

where

$$
\begin{aligned}
E(n)= & \int_{\Omega}\left(\left|\nabla T_{k}\left(u_{n}\right)\right|^{p(x)-2} \nabla T_{k}\left(u_{n}\right)\right. \\
& \left.\quad-\left|\nabla T_{k}(u)\right|^{p(x)-2} \nabla T_{k}(u)\right) \cdot \nabla\left(T_{k}\left(u_{n}\right)-T_{k}(u)\right) d x .
\end{aligned}
$$

We recall the following well-known inequalities: for any two real vectors $a, b \in$ $\mathbb{R}^{N}$,

$$
\left(a|a|^{p-2}-b|b|^{p-2}\right)(a-b) \geq c(p)|a-b|^{p} \quad \text { if } p \geq 2
$$

and for every $\varepsilon \in(0,1]$,

$$
|a-b|^{p} \leq c(p) \varepsilon^{(p-2) / p}\left(a|a|^{p-2}-b|b|^{p-2}\right)(a-b)+\varepsilon|b|^{p} \quad \text { if } 1<p<2,
$$

where $c(p)=\left(2^{1-p} / p-1\right)$ when $p \geq 2$ and $c(p)=\left(3^{2-p} / p-1\right)$ when $1<p<2$.

Thus

$$
\int_{\{x \in \Omega: p(x) \geq 2\}}\left|\nabla T_{k}\left(u_{n}\right)-\nabla T_{k}(u)\right|^{p(x)} d x \leq 2^{p_{+}-1}\left(p_{+}-1\right) E(n)
$$

and

$$
\begin{aligned}
& \int_{\{x \in \Omega: 1<p(x)<2\}}\left|\nabla T_{k}\left(u_{n}\right)-\nabla T_{k}(u)\right|^{p(x)} d x \\
& \quad \leq \frac{3^{2-p_{-}}}{p_{-}-1} \cdot \varepsilon^{\left(p_{-}-2\right) / p_{-}} E(n)+\varepsilon \int_{\Omega}\left|\nabla T_{k}(u)\right|^{p(x)} d x .
\end{aligned}
$$

Since $E(n) \rightarrow 0$ as $n \rightarrow+\infty$, then using the arbitrariness of $\varepsilon$ and the fact that $\nabla T_{k}(u)$ is bounded in $\left(L^{p(\cdot)}(\Omega)\right)^{N}$, we conclude that

$$
\lim _{n \rightarrow+\infty} \int_{\Omega}\left|\nabla T_{k}\left(u_{n}\right)-\nabla T_{k}(u)\right|^{p(x)} d x=0,
$$

which implies that, for every positive integer $k$,

$$
T_{k}\left(u_{n}\right) \rightarrow T_{k}(u) \quad \text { strongly in } W_{0}^{1, p(\cdot)}(\Omega) .
$$

Moreover, for every positive real number $r$, there exists a positive integer $k$ such that $r \leq k$. Recalling the fact that $T_{r}\left(T_{k}(\cdot)\right)=T_{r}(\cdot)$ and that

$$
T_{r}\left(T_{k}\left(u_{n}\right)\right) \rightarrow T_{r}\left(T_{k}(u)\right) \quad \text { strongly in } W_{0}^{1, p(\cdot)}(\Omega),
$$


we have, for every $r>0$,

$$
T_{r}\left(u_{n}\right) \rightarrow T_{r}(u) \quad \text { strongly in } W_{0}^{1, p(\cdot)}(\Omega),
$$

which also implies that

$$
\left|\nabla T_{r}\left(u_{n}\right)\right|^{p(x)-2} \nabla T_{r}\left(u_{n}\right) \rightarrow\left|\nabla T_{r}(u)\right|^{p(x)-2} \nabla T_{r}(u) \quad \text { in }\left(L^{p^{\prime}(\cdot)}(\Omega)\right)^{N} .
$$

Step 4. Show that $u$ is an entropy solution.

Now we choose $v_{n}=T_{k}\left(u_{n}-\phi\right)$ as a test function in (3.2) for $k>0$ and $\phi \in$ $W_{0}^{1, p(\cdot)}(\Omega) \cap L^{\infty}(\Omega)$. We note that, if $L=k+\|\phi\|_{L^{\infty}(\Omega)}$, then

$$
\begin{aligned}
& \int_{\Omega}\left|\nabla u_{n}\right|^{p(x)-2} \nabla u_{n} \cdot \nabla T_{k}\left(u_{n}-\phi\right) d x \\
& \quad=\int_{\Omega}\left|\nabla T_{L}\left(u_{n}\right)\right|^{p(x)-2} \nabla T_{L}\left(u_{n}\right) \cdot \nabla T_{k}\left(u_{n}-\phi\right) d x
\end{aligned}
$$

and

$$
\begin{aligned}
\int_{\Omega}\left|\nabla T_{L}\left(u_{n}\right)\right|^{p(x)-2} \nabla T_{L}\left(u_{n}\right) \cdot \nabla T_{k}\left(u_{n}-\phi\right) d x \\
\quad=\int_{\Omega} f_{n} T_{k}\left(u_{n}-\phi\right) d x+\int_{\Omega} F_{n} \cdot \nabla T_{k}\left(u_{n}-\phi\right) d x .
\end{aligned}
$$

Using (3.5) and (3.13), we pass to the limits as $n$ tends to infinity to conclude that

$$
\int_{\Omega}|\nabla u|^{p(x)-2} \nabla u \cdot \nabla T_{k}(u-\phi) d x=\int_{\Omega} f T_{k}(u-\phi) d x+\int_{\Omega} F \cdot \nabla T_{k}(u-\phi) d x,
$$

for every $k>0$ and every $\phi \in W_{0}^{1, p(\cdot)}(\Omega) \cap L^{\infty}(\Omega)$. This finishes the proof of the existence of entropy solutions.

(2) Uniqueness of entropy solutions. Suppose that $v$ is another entropy solution of problem (1.1); we will show that $u=v$ a.e. in $\Omega$. We write the entropy equality (1.4) corresponding to solution $u$ with test function $T_{h}(v)$ and $v$ with test function $T_{h}(u)$. Adding up both equalities, we find that

$$
\begin{gathered}
\int_{\left\{\left|u-T_{h}(v)\right| \leq k\right\}}\left[|\nabla u|^{p(x)-2} \nabla u-F\right] \cdot \nabla T_{k}\left(u-T_{h}(v)\right) d x \\
+\int_{\left\{\left|v-T_{h}(u)\right| \leq k\right\}}\left[|\nabla v|^{p(x)-2} \nabla v-F\right] \cdot \nabla T_{k}\left(v-T_{h}(u)\right) d x \\
\quad=\int_{\Omega} f\left[T_{k}\left(u-T_{h}(v)\right)+T_{k}\left(v-T_{h}(u)\right)\right] d x .
\end{gathered}
$$

With similar arguments as in [11,32] by some modifications, after discarding some nonnegative but uninteresting terms and passing to the limits in (3.14) as $h \rightarrow \infty$, we can reach the conclusion. Therefore, we complete the proof of Theorem 1.4. 
REMARK 3.1. We note that it is not obvious that the entropy solution $u$ of problem (1.1) can be a weak solution. However, if $\mu \in W^{-1, p^{\prime}(\cdot)}(\Omega)$ or $\mu \in L^{m(\cdot)}(\Omega), m(\cdot)=$ $\left(p^{*}(\cdot)\right)^{\prime}$, then $u \in W_{0}^{1, p(\cdot)}(\Omega)$. Under the above assumptions, the existence and uniqueness of weak solutions for problem (1.1) is a straightforward generalization of [18].

Next, we prove that the entropy solution $u$ is also a renormalized solution of problem (1.1) and the renormalized solution is unique.

PROOF OF THEOREM 1.5. (1) The entropy solution is a renormalized solution. First we observe that the entropy solution $u$ in Theorem 1.4 satisfies the requirement that $T_{k}(u) \in W_{0}^{1, p(\cdot)}(\Omega)$, for given $k>0$. Choosing $a=1$ in Proposition 2.7, we obtain the renormalized condition, that is,

$$
\lim _{k \rightarrow \infty} \int_{\{k \leq|u| \leq k+1\}}|\nabla u|^{p(x)} d x=0 .
$$

Let $\left\{u_{n}\right\}$ be a sequence constructed in (3.2), which satisfies the requirement that $T_{k}\left(u_{n}\right)$ strongly converges to $T_{k}(u)$ in $W_{0}^{1, p(x)}(\Omega)$, for every $k>0$.

Let $S \in W^{1, \infty}(\mathbb{R})$ be such that $\operatorname{supp} S \subset[-M, M]$ for some $M>0$. For every $\varphi \in C_{0}^{\infty}(\Omega), S\left(u_{n}\right) \varphi \in W_{0}^{1, p(x)}(\Omega)$ is a test function in (3.2). It yields

$$
\begin{gathered}
\int_{\Omega}\left[S\left(u_{n}\right)\left|\nabla u_{n}\right|^{p(x)-2} \nabla u_{n} \cdot \nabla \varphi+S^{\prime}\left(u_{n}\right)\left|\nabla u_{n}\right|^{p(x)} \varphi\right] d x \\
=\int_{\Omega}\left[f_{n} S\left(u_{n}\right) \varphi+F_{n} \cdot \nabla\left(S\left(u_{n}\right) \varphi\right)\right] d x .
\end{gathered}
$$

For the left-hand side of (3.15), because supp $S^{\prime} \subset[-M, M]$ we know that

$$
S\left(u_{n}\right)\left|\nabla u_{n}\right|^{p(x)-2} \nabla u_{n}=S\left(u_{n}\right)\left|\nabla T_{M}\left(u_{n}\right)\right|^{p(x)-2} \nabla T_{M}\left(u_{n}\right)
$$

and

$$
S^{\prime}\left(u_{n}\right)\left|\nabla u_{n}\right|^{p(x)}=S^{\prime}\left(u_{n}\right)\left|\nabla T_{M}\left(u_{n}\right)\right|^{p(x)} .
$$

Using (3.4), (3.12) and (3.13),

$$
S\left(u_{n}\right)\left|\nabla T_{M}\left(u_{n}\right)\right|^{p(x)-2} \nabla T_{M}\left(u_{n}\right) \rightarrow S(u)\left|\nabla T_{M}(u)\right|^{p(x)-2} \nabla T_{M}(u) \quad \text { in }\left(L^{p^{\prime}(\cdot)}(\Omega)\right)^{N}
$$

and

$$
S^{\prime}\left(u_{n}\right)\left|\nabla T_{M}\left(u_{n}\right)\right|^{p(x)} \rightarrow S^{\prime}(u)\left|\nabla T_{M}(u)\right|^{p(x)} \quad \text { in } L^{1}(\Omega) .
$$

Noting that

$$
\begin{gathered}
S(u)\left|\nabla T_{M}(u)\right|^{p(x)-2} \nabla T_{M}(u)=S(u)|\nabla u|^{p(x)-2} \nabla u, \\
S^{\prime}(u)\left|\nabla T_{M}(u)\right|^{p(x)}=S^{\prime}(u)|\nabla u|^{p(x)},
\end{gathered}
$$


we deduce that

$$
S\left(u_{n}\right)\left|\nabla u_{n}\right|^{p(x)-2} \nabla u_{n} \rightarrow S(u)|\nabla u|^{p(x)-2} \nabla u \quad \text { in }\left(L^{p^{\prime}(\cdot)}(\Omega)\right)^{N}
$$

and

$$
S^{\prime}\left(u_{n}\right)\left|\nabla u_{n}\right|^{p(x)} \rightarrow S^{\prime}(u)|\nabla u|^{p(x)} \quad \text { in } L^{1}(\Omega) .
$$

For the right-hand side of (3.15), since $\nabla\left[S\left(u_{n}\right) \varphi\right]=\nabla\left[S\left(T_{M}\left(u_{n}\right)\right) \varphi\right]$, thanks to the strong convergence of $T_{M}\left(u_{n}\right), f_{n}$ and $F_{n}$, it is easy to pass to the limits in the terms on the right-hand side. Therefore, we obtain

$$
\begin{gathered}
\int_{\Omega}\left[S(u)|\nabla u|^{p(x)-2} \nabla u \cdot \nabla \varphi+S^{\prime}(u)|\nabla u|^{p(x)} \varphi\right] d x \\
=\int_{\Omega}[f S(u) \varphi+F \cdot \nabla(S(u) \varphi)] d x,
\end{gathered}
$$

which is (ii) in Definition 1.3. This completes the proof of the existence of renormalized solutions.

(2) Uniqueness of renormalized solutions. Now we prove the uniqueness of renormalized solutions for problem (1.1) by choosing an appropriate test function. Let $u$ and $v$ be two renormalized solutions for problem (1.1). Fix a positive number $k$. For $\sigma>0$, let $S_{\sigma}$ be the function defined by

$$
\begin{cases}S_{\sigma}(r)=r & \text { if }|r|<\sigma, \\ S_{\sigma}(r)=\left(\sigma+\frac{1}{2}\right) \mp \frac{1}{2}(r \mp(\sigma+1))^{2} & \text { if } \sigma \leq \pm r \leq \sigma+1, \\ S_{\sigma}(r)= \pm\left(\sigma+\frac{1}{2}\right) & \text { if } \pm r>\sigma+1 .\end{cases}
$$

It is obvious that

$$
\begin{cases}S_{\sigma}^{\prime}(r)=1 & \text { if }|r|<\sigma \\ S_{\sigma}^{\prime}(r)=\sigma+1-|r| & \text { if } \sigma \leq|r| \leq \sigma+1 \\ S_{\sigma}^{\prime}(r)=0 & \text { if }|r|>\sigma+1\end{cases}
$$

It is easy to check $S_{\sigma} \in W^{2, \infty}(\mathbb{R})$ with supp $S_{\sigma}^{\prime} \subset[-\sigma-1, \sigma+1]$ and supp $S_{\sigma}^{\prime \prime} \subset$ $[\sigma, \sigma+1] \cup[-\sigma-1,-\sigma]$. Therefore, we may take $S=S_{\sigma}^{\prime}$ in (1.5) to have

$$
\begin{gathered}
\int_{\Omega}\left[S_{\sigma}^{\prime}(u)|\nabla u|^{p(x)-2} \nabla u \cdot \nabla \varphi+S_{\sigma}^{\prime \prime}(u)|\nabla u|^{p(x)} \varphi\right] d x \\
=\int_{\Omega}\left[f S_{\sigma}^{\prime}(u) \varphi+F \cdot \nabla\left(S_{\sigma}^{\prime}(u) \varphi\right)\right] d x
\end{gathered}
$$

and

$$
\begin{gathered}
\int_{\Omega}\left[S_{\sigma}^{\prime}(v)|\nabla v|^{p(x)-2} \nabla v \cdot \nabla \varphi+S_{\sigma}^{\prime \prime}(v)|\nabla v|^{p(x)} \varphi\right] d x \\
=\int_{\Omega}\left[f S_{\sigma}^{\prime}(v) \varphi+F \cdot \nabla\left(S_{\sigma}^{\prime}(v) \varphi\right)\right] d x .
\end{gathered}
$$


As $T_{k}\left(S_{\sigma}(u)-S_{\sigma}(v)\right) \in W_{0}^{1, p(\cdot)}(\Omega) \cap L^{\infty}(\Omega)$, we plug $\varphi=T_{k}\left(S_{\sigma}(u)-S_{\sigma}(v)\right)$ as a test function in the above equalities and subtract them to deduce that

$$
J_{1}+J_{2}=J_{3}+J_{4}+J_{5}
$$

where

$$
\begin{aligned}
J_{1} & =\int_{\Omega}\left(S_{\sigma}^{\prime}(u)|\nabla u|^{p(x)-2} \nabla u-S_{\sigma}^{\prime}(v)|\nabla v|^{p(x)-2} \nabla v\right) \cdot \nabla T_{k}\left(S_{\sigma}(u)-S_{\sigma}(v)\right) d x, \\
J_{2} & =\int_{\Omega}\left[S_{\sigma}^{\prime \prime}(u)|\nabla u|^{p(x)}-S_{\sigma}^{\prime \prime}(v)|\nabla v|^{p(x)}\right] T_{k}\left(S_{\sigma}(u)-S_{\sigma}(v)\right) d x, \\
J_{3} & =\int_{\Omega} f\left(S_{\sigma}^{\prime}(u)-S_{\sigma}^{\prime}(v)\right) T_{k}\left(S_{\sigma}(u)-S_{\sigma}(v)\right) d x, \\
J_{4} & =\int_{\Omega} T_{k}\left(S_{\sigma}(u)-S_{\sigma}(v)\right) F \cdot \nabla\left(S_{\sigma}^{\prime}(u)-S_{\sigma}^{\prime}(v)\right) d x, \\
J_{5} & =\int_{\Omega} F\left(S_{\sigma}^{\prime}(u)-S_{\sigma}^{\prime}(v)\right) \cdot \nabla T_{k}\left(S_{\sigma}(u)-S_{\sigma}(v)\right) d x .
\end{aligned}
$$

We estimate $J_{1}, J_{2}$ and $J_{3}$ one by one. Writing

$$
\begin{aligned}
J_{1}=\int_{\Omega} & {\left[\left|\nabla S_{\sigma}(u)\right|^{p(x)-2} \nabla S_{\sigma}(u)\right.} \\
& \left.-\left|\nabla S_{\sigma}(v)\right|^{p(x)-2} \nabla S_{\sigma}(v)\right] \cdot \nabla T_{k}\left(S_{\sigma}(u)-S_{\sigma}(v)\right) d x \\
& +\int_{\Omega}\left[S_{\sigma}^{\prime}(u)-S_{\sigma}^{\prime}(u)\left|S_{\sigma}^{\prime}(u)\right|^{p(x)-2}\right]|\nabla u|^{p(x)-2} \nabla u \cdot \nabla T_{k}\left(S_{\sigma}(u)-S_{\sigma}(v)\right) d x \\
& -\int_{\Omega}\left[S_{\sigma}^{\prime}(v)-S_{\sigma}^{\prime}(v)\left|S_{\sigma}^{\prime}(v)\right|^{p(x)-2}\right]|\nabla v|^{p(x)-2} \nabla v \cdot \nabla T_{k}\left(S_{\sigma}(u)-S_{\sigma}(v)\right) d x \\
:=J_{1}^{1} & +J_{1}^{2}+J_{1}^{3},
\end{aligned}
$$

and setting $\sigma \geq k$,

$$
J_{1}^{1} \geq \int_{\{|u-v| \leq k\} \cap\{|u|,|v| \leq k\}}\left(|\nabla u|^{p(x)-2} \nabla u-|\nabla v|^{p(x)-2} \nabla v\right) \cdot \nabla(u-v) d x .
$$

Recalling that $\operatorname{supp} S_{\sigma}^{\prime} \subset[-\sigma-1, \sigma+1]$ and $\operatorname{supp} S_{\sigma}^{\prime \prime} \subset[\sigma, \sigma+1] \cup[-\sigma-1,-\sigma]$, we obtain

$$
\begin{aligned}
\left|J_{1}^{2}\right| \leq & \int_{\{\sigma \leq|u| \leq \sigma+1\}}|\nabla u|^{p(x)} d x \\
& +\int_{\{\sigma \leq|u| \leq \sigma+1\} \cap\{|v| \leq \sigma+1\} \cap\left\{\left|S_{\sigma}(u)-S_{\sigma}(v)\right| \leq k\right\}}|\nabla u|^{p(x)-1}|\nabla v| d x
\end{aligned}
$$




$$
\begin{aligned}
& \leq 2\left(\int_{\{\sigma \leq|u| \leq \sigma+1\}}|\nabla u|^{p(x)} d x\right. \\
& \left.\quad+\int_{\{\sigma \leq|u| \leq \sigma+1\} \cap\{\sigma-k \leq|v| \leq \sigma+1\}}|\nabla u|^{p(x)-1}|\nabla v| d x\right) \\
& \leq C\left(\int_{\{\sigma \leq|u| \leq \sigma+1\}}|\nabla u|^{p(x)} d x+\int_{\{\sigma-k \leq|v| \leq \sigma+1\}}|\nabla v|^{p(x)} d x\right) .
\end{aligned}
$$

Moreover, we may get the similar estimate for $J_{1}^{3}$. Furthermore,

$$
\left|J_{2}\right| \leq C\left(\int_{\{\sigma \leq|u| \leq \sigma+1\}}|\nabla u|^{p(x)} d x+\int_{\{\sigma \leq|v| \leq \sigma+1\}}|\nabla v|^{p(x)} d x\right) .
$$

From the above estimates and Definition 1.3(i), we obtain

$$
\lim _{\sigma \rightarrow \infty}\left(\left|J_{1}^{2}\right|+\left|J_{1}^{3}\right|+\left|J_{2}\right|\right)=0
$$

Observing that

$$
f\left(S_{\sigma}^{\prime}(u)-S_{\sigma}^{\prime}(v)\right) \rightarrow 0 \quad \text { strongly in } L^{1}(\Omega)
$$

as $\sigma \rightarrow \infty$ and using the Lebesgue dominated convergence theorem, we deduce that

$$
\lim _{\sigma \rightarrow \infty}\left|J_{3}\right|=0
$$

From Lemma 2.1 and $S_{\sigma} \in W^{2, \infty}(\mathbb{R})$,

$$
\begin{aligned}
\left|J_{4}\right| & \leq k \int_{\Omega}|F|\left(\left|S_{\sigma}^{\prime \prime}(u) \nabla u\right|+\left|S_{\sigma}^{\prime \prime}(v) \nabla v\right|\right) d x \\
& \leq k \int_{\{\sigma \leq|u| \leq \sigma+1\}}|F| \cdot|\nabla u| d x+\int_{\{\sigma \leq|v| \leq \sigma+1\}}|F| \cdot|\nabla v| d x \\
& \leq 2 k|F|_{p^{\prime}(\cdot)}\left(|\nabla u|_{p(\cdot),\{\sigma \leq|u| \leq \sigma+1\}}+|\nabla v|_{p(\cdot),\{\sigma \leq|v| \leq \sigma+1\}}\right) .
\end{aligned}
$$

By means of Lemma 2.2 and Definition 1.3(i), we obtain

$$
\lim _{\sigma \rightarrow \infty}\left|J_{4}\right|=0
$$

Next we show that

$$
\lim _{\sigma \rightarrow \infty}\left|J_{5}\right|=0
$$

Write

$$
\begin{aligned}
\left|J_{5}\right| & \leq \int_{\Omega}|F|\left|S_{\sigma}^{\prime}(u)-S_{\sigma}^{\prime}(v)\right| T_{k}^{\prime}\left(S_{\sigma}(u)-S_{\sigma}(v)\right)\left|S_{\sigma}^{\prime}(u) \nabla u-S_{\sigma}^{\prime}(v) \nabla v\right| d x \\
& =\int_{\Omega} G(u, v) d x .
\end{aligned}
$$


We divide the estimate of $J_{5}$ into several cases according to the different integration sets. Denote

$$
\begin{aligned}
& E_{1}=\{|u| \leq \sigma,|v| \leq \sigma\} \cup\{|u| \geq \sigma+1,|v| \geq \sigma+1\}, \\
& E_{2}=\{|u| \leq \sigma,|v| \geq \sigma+1\} \cup\{|v| \leq \sigma,|u| \geq \sigma+1\}=E_{21} \cup E_{22}, \\
& E_{3}=\{|u| \leq \sigma, \sigma \leq|v| \leq \sigma+1\} \cup\{|v| \leq \sigma, \sigma \leq|u| \leq \sigma+1\}=E_{31} \cup E_{32}, \\
& E_{4}=\{\sigma \leq|u| \leq \sigma+1, \sigma \leq|v| \leq \sigma+1\} \\
& E_{5}=\{\sigma \leq|u| \leq \sigma+1,|v| \geq \sigma+1\} \cup\{\sigma \leq|v| \leq \sigma+1,|u| \geq \sigma+1\} .
\end{aligned}
$$

It is obvious that $\Omega=E_{1} \cup E_{2} \cup E_{3} \cup E_{4} \cup E_{5}$.

From the definition of $S_{\sigma}$ and supp $S_{\sigma}^{\prime} \subset[-\sigma-1, \sigma+1]$, we deduce the following estimates.

Estimate on $E_{1}$.

$$
\int_{E_{1}} G(u, v) d x=0
$$

Estimate on $E_{2}$.

$$
\begin{aligned}
\int_{E_{21}} G(u, v) d x & \leq \int_{\left\{\left|S_{\sigma}(u)-S_{\sigma}(v)\right| \leq k\right\} \cap\{|u| \leq \sigma\} \cap\{|v| \geq \sigma+1\}}|F||\nabla u| d x \\
& \leq \int_{\{\sigma-k \leq|u| \leq \sigma\}}|F||\nabla u| d x
\end{aligned}
$$

and

Estimate on $E_{3}$.

$$
\int_{E_{22}} G(u, v) d x \leq \int_{\{\sigma-k \leq|v| \leq \sigma\}}|F||\nabla v| d x .
$$

$$
\begin{aligned}
\int_{E_{31}} G(u, v) d x & \leq \int_{\left\{\left|S_{\sigma}(u)-S_{\sigma}(v)\right| \leq k\right\} \cap\{|u| \leq \sigma\} \cap\{\sigma \leq|v| \leq \sigma+1\}} G(u, v) d x \\
& \leq 2\left(\int_{\{\sigma-k \leq|u| \leq \sigma\}}|F||\nabla u| d x+\int_{\{\sigma-k \leq|v| \leq \sigma+1\}}|F||\nabla v| d x\right)
\end{aligned}
$$

and

$$
\int_{E_{32}} G(u, v) d x \leq 2\left(\int_{\{\sigma-k \leq|u| \leq \sigma+1\}}|F||\nabla u| d x+\int_{\{\sigma-k \leq|v| \leq \sigma\}}|F||\nabla v| d x\right) .
$$

Estimate on $E_{4}$.

$$
\int_{E_{4}} G(u, v) d x \leq 2\left(\int_{\{\sigma \leq|u| \leq \sigma+1\}}|F||\nabla u| d x+\int_{\{\sigma \leq|v| \leq \sigma+1\}}|F||\nabla v| d x\right) .
$$

Estimate on $E_{5}$.

$$
\int_{E_{5}} G(u, v) d x \leq \int_{\{\sigma \leq|u| \leq \sigma+1\}}|F||\nabla u| d x+\int_{\{\sigma \leq|v| \leq \sigma+1\}}|F||\nabla v| d x .
$$


Summing up the above estimates,

$$
\begin{aligned}
\left|J_{5}\right| & \leq \int_{\Omega} G(u, v) d x \\
& \leq C\left(\int_{\{\sigma-k \leq|u| \leq \sigma+1\}}|F||\nabla u| d x+\int_{\{\sigma-k \leq|v| \leq \sigma+1\}}|F||\nabla v| d x\right),
\end{aligned}
$$

which implies from Lemmas 2.1, 2.2 and Definition 1.3(i) that

$$
\lim _{\sigma \rightarrow \infty}\left|J_{5}\right|=0
$$

Therefore, sending $\sigma \rightarrow \infty$ in (3.17) and recalling (3.18),

$$
\int_{\{|u| \leq k / 2,|v| \leq k / 2\}}\left(|\nabla u|^{p(x)-2}-|\nabla v|^{p(x)-2} \nabla v\right) \cdot \nabla(u-v) d x=0,
$$

which implies $\nabla u=\nabla v$ a.e. on the set $\{|u| \leq k / 2,|v| \leq k / 2\}$. Since $k$ is arbitrary, we conclude that $\nabla u=\nabla v$ a.e. in $\Omega$.

Moreover, it follows from Lemma 2.4 that

$$
\left|T_{k}(u-v)\right|_{p(x)} \leq C\left|\nabla T_{k}(u-v)\right|_{p(x)}=0 \quad \text { for all } k>0,
$$

and hence $u=v$ a.e. in $\Omega$. Therefore, we obtain the uniqueness of renormalized solutions. This completes the proof of Theorem 1.5.

\section{Extensions}

The existence results above of the entropy solutions and renormalized solutions can be extended to the general problem

$$
\begin{cases}-\operatorname{div}(a(x, u, \nabla u)+\Phi(u))=\mu & \text { in } \Omega \\ u=0 & \text { on } \partial \Omega\end{cases}
$$

where $\mu$ is a signed measure in $L^{1}(\Omega)+W^{-1, p^{\prime}(\cdot)}(\Omega), a: \Omega \times \mathbb{R} \times \mathbb{R}^{N} \rightarrow \mathbb{R}^{N}$ is a Carathéodory function such that, for every $s \in \mathbb{R}$, we have $\xi, \eta \in \mathbb{R}^{N}$ and, for almost every $x \in \Omega$,

$$
\begin{gathered}
a(x, s, \xi) \cdot \xi \geq \lambda|\xi|^{p(x)}, \\
|a(x, s, \xi)| \leq b(|s|)\left(c(x)+d|\xi|^{p(x)-1}\right), \\
(a(x, s, \xi)-a(x, s, \eta)) \cdot(\xi-\eta)>0, \quad \text { where } \xi \neq \eta .
\end{gathered}
$$

Herein, $p_{-}>1, \lambda>0, d \in \mathbb{R}, c(x) \in L^{p^{\prime}(\cdot)}(\Omega)$ and $b:[0,+\infty) \rightarrow(0,+\infty), \Phi$ : $\mathbb{R} \rightarrow \mathbb{R}^{N}$ are continuous functions.

When $p(x)$ is a constant, we know from [11] that $\mu \in L^{1}(\Omega)+W^{-1, p^{\prime}}(\Omega)$ if and only if $\mu \in \mathcal{M}_{b}^{p}(\Omega)$, that is, every signed measure that is zero on the sets of zero 
$p$-capacity can be decomposed into the sum of a function in $L^{1}(\Omega)$ and an element in $W^{-1, p^{\prime}}(\Omega)$, and conversely, every signed measure in $L^{1}(\Omega)+W^{-1, p^{\prime}}(\Omega)$ has zero measure for the sets of zero $p$-capacity. For the variable exponent case, using the same arguments as in [11], we feel that the similar decomposition result should be true by the properties of $L^{p(\cdot)}(\Omega)$ and the relative $p(\cdot)$-capacity (see [22]), but this extension remains open.

\section{References}

[1] E. Acerbi and G. Mingione, 'Regularity results for a class of functionals with non-standard growth', Arch. Ration. Mech. Anal. 156 (2001), 121-140.

[2] E. Acerbi and G. Mingione, 'Regularity results for stationary electro-rheological fluids', Arch. Ration. Mech. Anal. 164 (2002), 213-259.

[3] E. Acerbi, G. Mingione and G. A. Seregin, 'Regularity results for parabolic systems related to a class of non Newtonian fluids', Ann. Inst. H. Poincaré Anal. Non Linéaire 21 (2004), 25-60.

[4] A. Alvino, L. Boccardo, V. Ferone, L. Orsina and G. Trombetti, 'Existence results for nonlinear elliptic equations with degenerate coercivity', Ann. Mat. Pura Appl. 182 (2003), 53-79.

[5] S. N. Antontsev and S. I. Shmarev, 'A model porous medium equation with variable exponent of nonlinearity: existence, uniqueness and localization properties of solutions', Nonlinear Anal. $\mathbf{6 0}$ (2005), 515-545.

[6] P. Bénilan, L. Boccardo, T. Gallouët, R. Gariepy, M. Pierre and J. L. Vazquez, 'An $L^{1}$-theory of existence and uniqueness of solutions of nonlinear elliptic equations', Ann. Sc. Norm. Super. Pisa Cl. Sci. 22 (1995), 241-273.

[7] D. Blanchard and F. Murat, 'Renormalised solutions of nonlinear parabolic problems with $L^{1}$ data: existence and uniqueness', Proc. Roy. Soc. Edinburgh Sect. A 127(6) (1997), 1137-1152.

[8] D. Blanchard, F. Murat and H. Redwane, 'Existence and uniqueness of a renormalized solution for a fairly general class of nonlinear parabolic problems', J. Differential Equations 177(2) (2001), 331-374.

[9] D. Blanchard and H. Redwane, 'Renormalized solutions for a class of nonlinear evolution problems', J. Math. Pure Appl. 77 (1998), 117-151.

[10] L. Boccardo and G. R. Cirmi, 'Existence and uniqueness of solution of unilateral problems with $L^{1}$ data', J. Convex. Anal. 6 (1999), 195-206.

[11] L. Boccardo, T. Gallouët and L. Orsina, 'Existence and uniqueness of entropy solutions for nonlinear elliptic equations with measure data', Ann. Inst. H. Poincaré Anal. Non Linéaire 13(5) (1996), 539-551.

[12] L. Boccardo, D. Giachetti, J. I. Diaz and F. Murat, 'Existence and regularity of renormalized solutions for some elliptic problems involving derivations of nonlinear terms', J. Differential Equations 106 (1993), 215-237.

[13] Y. Chen, S. Levine and M. Rao, 'Variable exponent, linear growth functionals in image restoration', SIAM J. Appl. Math. 66 (2006), 1383-1406.

[14] L. Diening, 'Riesz potential and Sobolev embeddings on generalized Lebesgue and Sobolev spaces $L^{p(\cdot)}$ and $W^{k, p(\cdot)}$, Math. Nachr. 268 (2004), 31-43.

[15] R. J. DiPerna and P. L. Lions, 'On the Cauchy problem for Boltzmann equations: global existence and weak stability', Ann. of Math. (2) 130 (1989), 321-366.

[16] X. Fan, 'Global $C^{1, \alpha}$ regularity for variable exponent elliptic equations in divergence form', J. Differential Equations 235 (2007), 397-417.

[17] X. Fan, J. Shen and D. Zhao, 'Sobolev embedding theorems for spaces $W^{k, p(x)}(\Omega)$ ', J. Math. Anal. Appl. 262 (2001), 749-760.

[18] X. Fan and Q. Zhang, 'Existence of solutions for $p(x)$-Laplacian Dirichlet problem', Nonlinear Anal. 52 (2003), 1843-1852.

[19] X. Fan, Q. Zhang and D. Zhao, 'Eigenvalues of $p(x)$-Laplacian Dirichlet problem', J. Math. Anal. Appl. 302 (2005), 306-317. 
[20] X. Fan and D. Zhao, 'On the spaces $L^{p(x)}(\Omega)$ and $W^{m, p(x)}(\Omega)^{\prime}$, J. Math. Anal. Appl. 263 (2001), 424-446.

[21] P. Harjulehto, 'Variable exponent Sobolev spaces with zero boundary values', Math. Bohem. 132 (2007), 125-136.

[22] P. Harjulehto, P. Hästö and M. Koskenoja, 'Properties of capacities in variable exponents Sobolev spaces', J. Anal. Appl. 5(2) (2007), 71-92.

[23] P. Harjulehto, P. Hästö, M. Koskenoja and S. Varonen, 'The Dirichlet energy integral and variable exponent Sobolev spaces with zero boundary values', Potential Anal. 25(3) (2006), 205-222.

[24] O. Kováčik and J. Rákosník, 'On spaces $L^{p}(x)$ and $W^{k, p(x)}$ ', Czechoslovak Math. J. 41(116) (1991), 592-618.

[25] C. Leone and A. Porretta, 'Entropy solutions for nonlinear elliptic equations in $L^{1}$, Nonlinear Anal. 32(3) (1998), 325-334.

[26] J. L. Lions, Quelques méthodes de résolution des problèmes aux limites non linéaire (Dunod et Gauthier Villars, Paris, 1969).

[27] P. L. Lions, Mathematical Topics in Fluid Mechanics, Incompressible Models, 1 (Oxford University Press, Oxford, 1996).

[28] J. Musielak, Orlicz Spaces and Modular Spaces (Springer, Berlin, 1983).

[29] M. C. Palmeri, 'Entropy subsolutions and supersolutions for nonlinear elliptic equations in $L^{1}$, Ric. Mat. 53 (2004), 183-212.

[30] K. Rajagopal and M. Rủžička, 'Mathematical modelling of electro-rheological fluids', Contin. Mech. Thermodyn. 13 (2001), 59-78.

[31] M. Rủžička, Electrorheological Fluids: Modeling and Mathematical Theory, Lecture Notes in Mathematics, 1748 (Springer, Berlin, 2000).

[32] M. Sanchón and J. M. Urbano, 'Entropy solutions for the $p(x)$-Laplace equation', Trans. Amer. Math. Soc. 361 (2009), 6387-6405.

[33] V. V. Zhikov, 'On some variational problems', Russ. J. Math. Phys. 5 (1997), 105-116.

[34] V. V. Zhikov, 'On the density of smooth functions in Sobolev-Orlicz spaces', Zap. Nauchn. Sem. S.-Peterburg. Otdel. Mat. Inst. Steklov. (POMI) 226 (2004), 67-81.

CHAO ZHANG, LMAM, School of Mathematical Sciences, Peking University, Beijing 100871, PR China

e-mail: czhang@math.pku.edu.cn

SHULIN ZHOU, LMAM, School of Mathematical Sciences, Peking University, Beijing 100871, PR China

e-mail: szhou@math.pku.edu.cn 